\title{
Saúde pública e terapia ocupacional: apontamentos sobre relações históricas e atuais
}

\author{
Public health and occupational therapy: \\ appointments on current and historical relations
}

\author{
Ana Paula Serrata Malfitano ${ }^{1}$, Ana Paula Ferreira ${ }^{2}$
}

\begin{abstract}
MALFITANO, A. P. S., FERREIRA, A. P. Saúde pública e terapia ocupacional: apontamentos sobre relações históricas e atuais. Rev. Ter. Ocup. Univ. São Paulo, v. 22, n. 2, p. 102-109, maio/ ago. 2011.
\end{abstract}

\begin{abstract}
RESUMO: Afirmam-se o enraizamento e a influência das políticas de saúde pública brasileira no desenvolvimento socio-histórico da profissão terapia ocupacional. Para tanto, parte-se da trajetória histórico-política das duas áreas e suas institucionalizações, num percurso da filantropia e assistencialismo ao direito social, alcançado a partir do movimento de democratização do Brasil do final da década de 1980, que ampliou os direitos sociais para todos. Como um dos resultados deu-se a implementação do Sistema Único de Saúde (SUS), que prevê o atendimento para todos e, portanto, a inclusão de grupos populacionais até então assistidos prioritariamente nos serviços filantrópicos. Essa necessidade criou a demanda por profissionais até então escassos no sistema público, como terapeutas ocupacionais. Desde então, técnicos têm criado novos serviços e tecnologias de atenção e cuidado que possibilitaram a ainda precária inserção de determinados grupos no acesso ao sistema de saúde e, consequentemente, o desenvolvimento da categoria profissional em questão. Por esse percurso, afirmamos que, por meio do estabelecimento de políticas públicas que expandam o acesso aos direitos sociais dos grupos-alvo da terapia ocupacional, podem-se ampliar, como consequência, os espaços de intervenção profissional e o desenvolvimento da categoria. Por fim, apontam-se as dificuldades atuais em torno da ordem político-econômica vigente e o permanente desafio de ampliação da atenção e inclusão de grupos populacionais no acesso aos direitos sociais.
\end{abstract}

DESCRITORES: Terapia ocupacional; Políticas públicas; Sistema Único de Saúde; Direito à saúde.

\footnotetext{
1. Terapeuta Ocupacional, Doutora em Saúde Pública, Professora Adjunta do Departamento de Terapia Ocupacional e do Programa de Pós-Graduação em Terapia Ocupacional da Universidade Federal de São Carlos (UFSCar). E-mail: anamalfitano@ufscar.br.

${ }^{2}$ Terapeuta Ocupacional, Residente do Programa "Residência Multiprofissional em Saúde da Família e Comunidade" da Universidade Federal de São Carlos (UFSCar). E-mail: paula5.fer@bol.com.br.

Endereço para correspondência: Universidade Federal de São Carlos. Departamento de Terapia Ocupacional - Laboratório METUIA. Rodovia Washington Luís, km. 235, São Carlos, SP. Caixa Postal 676. CEP: 13565-905. E-mail: anamalfitano@ufscar.br.
} 
Percurso histórico da implementação do SUS e do desenvolvimento profissional da terapia ocupacional: caminhos congruentes

$\mathrm{A}$ história de desenvolvimento da profissão terapia ocupacional enraíza-se em um trabalho calcado no assistencialismo e na caridade, com base no modelo da medicina hospitalar na Europa, no início do século XIX, quando as equipes de saúde tinham como objetivo realizar um trabalho caritativo, com o intuito de "salvar a alma do pobre e a sua própria" (DE CARLO; BARTALOTTI, 2001, p. 20).

O percurso da psiquiatria, por sua vez, influencia o desenvolvimento e o reconhecimento da profissão a partir do uso do trabalho e da ocupação como instrumentos de tratamento na instituição asilar (NASCIMENTO, 1991). No Brasil, essas modalidades de tratamento foram introduzidas pela vinda da família real portuguesa, com a instauração, entre outras ações, do Hospital D. Pedro II, em 1852, no Rio de Janeiro, mais tarde chamado Hospício Nacional de Alienados e, posteriormente, em 1898, do Hospital Colônia de Juqueri. A terapêutica pelo trabalho, o tratamento moral, foi influenciada pelo alienismo europeu - modelo utilizado para a organização dos serviços de assistência no Brasil - e apoiava-se na ideia de recuperação do louco por meio da atividade laboral. Pelo histórico estudado dessa modalidade de tratamento (FOUCAULT, 1979; BASAGLIA, 1986), podemos reafirmar que o tratamento moral foi utilizado também nos manicômios como forma de proteger a sociedade daqueles que ameaçavam às normas vigentes, aplicando o isolamento como modalidade de manutenção da ordem social. A relação de dominação, hierarquia, vigilância e ordem eram aspectos instituídos e avaliados como terapêuticos, sendo o trabalho visto como forma natural para instaurá-los com o intuito de reeducar e recompor os alienados mentais. Contudo, Nascimento (1991) constata que o trabalho estava mais à mercê da manutenção das instituições do que destinado ao caráter educativo, estabelecido pela incorporação de técnicas disciplinares e da regulação pela ordem.

É importante salientar que se embutiam, em tais formas de tratamento, os mecanismos disciplinares incorporados na sociedade industrial do século XX. Os alienados tinham todas as atividades controladas, confirmando a prática do poder disciplinar dentro das instituições manicomiais. Os internos eram privados de seus direitos, vivendo sob ameaças e violências, além de não exercerem qualquer poder sobre o trabalho que realizavam, o que certifica seu caráter de exploração e dominação. Foucault (1979) exemplifica as relações de poder adotadas nas instituições psiquiátricas:

O poder disciplinar apoia-se mais nos corpos e em seus atos do que na terra e seus produtos, para extrair dos corpos tempo e trabalho mais do que bens e riqueza. É um tipo de poder que se exerce continuamente através da vigilância (...) e que supõe mais de um sistema minucioso de coerções materiais do que a existência física de um soberano (p. 187).

Nesses locais, as atividades de ocupação estavam intrinsecamente ligadas à rotina dos manicômios e contavam com profissionais para sua coordenação. A terapia ocupacional inicia sua institucionalização por meio desses espaços e dessas atividades (SOARES, 2007).

No século XX, durante o curso da Primeira Guerra Mundial, a profissão é reconhecida nos Estados Unidos com a função social de reabilitação dos homens feridos nos combates, desenvolvendo serviços nos hospitais do Exército, contribuindo com o movimento nacional de auxílio à reconstrução nacional.

No Brasil, o reconhecimento legal da profissão recebeu forte influência norte-americana, sendo esta uma referência para a capacitação dos profissionais brasileiros, com uma imagem muito ligada à assistência a pessoas com problemas de ordem motora (neurológicos ou ortopédicos) devido à herança do movimento internacional de reabilitação (DE CARLO; BARTALOTTI, 2001). Em 13 de outubro de 1969, através do Decreto-Lei n$^{\circ}$ 938, são definidas as atribuições do terapeuta ocupacional e a formação de nível superior é reconhecida.

A legitimação e o início das ações da profissão inserem-se num momento particular da história brasileira, a ditadura militar, que certamente marca a saúde, em geral, e a terapia ocupacional, em particular.

Na saúde, a administração ocorria prioritariamente por meio do Instituto Nacional de Previdência Social (INPS), que operava em parceria com o setor privado para a oferta de atenção médica individual. $\mathrm{O}$ acesso à saúde pública era restrito aos trabalhadores que contribuíam financeiramente com parte de seus salários para o sistema previdenciário. Estabeleceu-se, desta maneira, a formação de polos: de um lado, o Estado, responsável pelas intervenções coletivas, e de outro, a assistência médica individual, oferecida pelo setor privado em parceria com o Estado aos trabalhadores formalmente registrados. Esta lógica criava um direito parcial de acesso à saúde, apenas àqueles que contribuíam para o sistema, estabelecendo uma "cidadania regulada", na qual se tem a estratificação de privilégios com base na condição de inserção no processo produtivo (ESCOREL, 1993). Mendes (1993) nomeia este modelo como "médico- 
assistencial-privatista", predominante nas décadas de 1970 e 1980, que reforçava a lógica de assistência médica de forma individual e organizada por um sistema privado contratado e conveniado com financiamento público. Tal modelo, predominantemente curativo, focava-se na demanda espontânea de cada cidadão, deixando sem suporte aqueles indivíduos que não acessavam os serviços públicos de saúde. Segundo Paim (1999, p.568), este é um modelo que foi "fortalecido com a expansão da previdência social e consolidado com a capitalização da medicina nas últimas décadas".

Grande parte da população brasileira, que até então não estava inserida no mercado de trabalho, obtinha os cuidados à saúde nos locais de caridade, como as Santas Casas de Misericórdia, sendo estas responsáveis, durante muito tempo, por grande parte dos cuidados com a saúde da maioria dos brasileiros.

A desigualdade de acesso à assistência à saúde, revelada pela configuração política do sistema público acima apresentada, não incluía a discussão sobre o atendimento específico a grupos portadores de deficiência física e/ou transtorno mental, embora tivesse diferença na história da assistência a esses grupos, alijando tais sujeitos para as instituições filantrópicas na busca de respostas às suas demandas. Esses grupos, alvo majoritário de ação dos terapeutas ocupacionais, encontravam-se no setor caritativo do sistema de saúde, e, consequentemente, era nesse espaço institucional que a categoria profissional em questão desenvolvia sua intervenção profissional. Dessa forma, as ações predominantes da profissão eram realizadas, quase exclusivamente, no setor filantrópico.

Outra característica que compõe a estrutura histórica da categoria dos terapeutas ocupacionais, enlaçada às intervenções no campo caritativo social, diz respeito à predominância de mulheres no exercício profissional. Diversos estudos de gênero discutem a função social do cuidado aliada às profissões de saúde, como enfermeiras e terapeutas ocupacionais, acrescida da visão da caridade e bondade presente em torno da população atendida (VERDÈS-LEROUX, 1986).

Esses fatores marcaram, e ainda marcam, o imaginário em torno das práticas dos terapeutas ocupacionais e revelam que os grupos para os quais dedicamos nossa intervenção eram, e ainda são, vistos pela dimensão da caridade e não pelos seus direitos.

Tal histórico corrobora com a estrutura da assistência à saúde, que excluiu esses sujeitos da esfera pública de atenção, implicando assim a permanência "apenas" dos trabalhadores, ou seja, os contribuintes do sistema previdenciário. A mudança na Constituição e legislação no final da década de 1980, durante o processo de redemocratização do país, alavancou a reconfiguração do lugar social daqueles sujeitos. Naquela ocasião, por ação dos movimentos sociais, ocorreram, entre outros movimentos, as conferências municipais e estaduais de saúde, que culminaram na realização da VIII Conferência Nacional de Saúde, em 1986, cujas diretrizes gerais determinaram a criação do Sistema Único de Saúde, SUS, em 1990. O advento da Constituição de 1988 trouxe consigo a consolidação, no plano legislativo, do reconhecimento dos direitos sociais, prevendo saúde para todos e como um dever do Estado. Posteriormente, em 1990, com o estabelecimento da lei 8080 , os princípios doutrinários do SUS (universalidade, integralidade e equidade) e as suas diretrizes organizativas (descentralização, regionalização, hierarquização) (BRASIL, 1990) apresentaram-se como a base para a reorganização do sistema, dos serviços, das práticas de saúde e, consequentemente, do paradigma em que se apoiou o ponto de vista defendido de processo saúde-doença. Assim, foi possível enunciar e defender a modificação do enfoque curativo dos serviços do sistema de saúde para a sua ampliação para as três esferas componentes da saúde: a promoção, a prevenção e a reabilitação.

Grupos como portadores de deficiência física e transtorno mental são, a partir desse marco, incluídos no atendimento em saúde pública, pois a saúde passa agora a se apresentar, legalmente, como um direito de todo e qualquer cidadão brasileiro, deixando para trás a concepção de um direito derivado do contrato de trabalho ou resultado de ações caritativas. Assim, para o sistema público de saúde, a incorporação desses sujeitos demarca também a ampliação das categorias profissionais necessárias para o funcionamento dos serviços, que iniciam experiências até então inovadoras, uma vez que aqueles sujeitos não ocupavam a arena pública de assistência à saúde. Essas experiências foram permitidas a partir de um novo espaço para a construção de novas ações em saúde e inclui a ampliação dos profissionais da terapia ocupacional nesse contexto.

\section{Obstáculos para avanços da saúde no panorama brasileiro}

A implantação do SUS, na década de 1990, aconteceu em meio a crescentes dificuldades políticoeconômicas, década na qual começava a ocorrer a inserção brasileira na nova ordem econômica mundial, denominada mundialização ou globalização, juntamente com um relevante processo interno de democratização e assunção dos direitos sociais, conforme destacamos acima. Hoje 
pode-se notar que nosso sistema de saúde está instalado em um contexto incongruente ao neoliberalismo, o qual prega a diminuição da participação do Estado na vida nacional, responsabilizando os indivíduos por si próprios, inclusive no âmbito dos cuidados com a saúde. A justificativa para a aceitação de tais ideias pela população brasileira foi pautada em um discurso de contenção de gastos sociais, na medida em que seria uma solução para amenizar a crise econômica instalada.

O programa neoliberal brasileiro foi concretizado nos governos de Fernando Collor de Mello e Fernando Henrique Cardoso, justamente no momento em que havia uma inusitada mudança no campo dos direitos, incluindo a saúde. A implementação do SUS, um sistema que denomina a saúde como direito universal e dever do Estado, depende de ações políticas para sua efetivação. A contradição criada pode ser refletida no questionamento de como implementar e manter um sistema público que prevê atenção para todos, sob responsabilidade do Estado, em um contexto de enxugamento de gastos.

Nessa ordem econômica, a diminuição dos investimentos sociais por parte do Estado faz-se condição, resultando num processo que gera um discurso e, consequentemente, um imaginário, que associa o Estado à ineficiência, e, inversamente, a iniciativa privada ao lema da eficiência, revelando as manifestações autoritárias do capitalismo, em que o lucro é sempre o principal objetivo, independentemente de razões humanistas (CARVALHO, 2000). De acordo com Laurell, citado por Carvalho (2000, p.8), no regime neoliberal "a atuação do Estado deve estar voltada para a garantia de um pacote mínimo de serviços essenciais voltado para os comprovadamente pobres, cabendo ao setor privado oferecer outras classes de serviços, de mais alto rendimento".

Essa visão de mundo se expandiu concomitantemente ao processo de globalização dos mercados mundiais, colaborando para o enfraquecimento de movimentos sociais referentes à defesa da saúde como um direito, o que, consequentemente, contribuiu para o processo da "universalização excludente", entendida como o princípio de universalização da saúde não realizado, ou seja, o não atendimento de todos no âmbito do SUS. Instala-se, portanto, o desmantelamento do sentido dado à saúde na Constituição de 1988, estando estreitamente ligado ao crescimento dos serviços médicos como bens privados, em contraposição ao direito universal, em que o Estado passa a realizar os serviços que não são interessantes à lógica privada (CARVALHO, 2000).

Dessa maneira, prevalece ainda o modelo liberalprivatista de atenção à saúde, regido pela lógica de mercado, com um caráter "predominantemente curativo, de atenção fragmentada e de baixo impacto sobre o nível de saúde da população" (CARVALHO, 2000, p. 11). Nesta lógica, mais uma vez, não há a priorização de grupos para os quais a terapia ocupacional, classicamente, dedica sua intervenção; pelo contrário, eles permanecem no sistema filantrópico. Contudo, a mudança do paradigma legislativo impulsiona outras discussões e a possibilidade de mudanças no cenário, tensionando a disputa entre direito social e ordem econômica.

O regime neoliberal freia, portanto, a mudança concreta e, assim também, a prevalência no imaginário da população concernente ao conceito de saúde, que deveria estar no patamar dos direitos, e não mais no da caridade, para todos. Dessa forma, prevalecem no Brasil as bases legislativas para a constituição de uma sociedade igualitária, com raízes na democracia plena, o que vale destacar que é de extrema relevância. Entretanto, a vontade política e a mobilização da sociedade civil são instrumentos fundamentais para que as políticas sociais, como o SUS, sejam implementadas, por isso tais ações vão, cada vez mais, na contramão dos valores econômicos vigentes, comprometendo, no caso da saúde, a possibilidade de construção de uma atenção mais qualitativa, universal, integral e equânime.

\section{Novas ações em saúde: a possibilidade de criação de experiências inovadoras}

Com a perspectiva do SUS no trabalho em saúde, que parte da inclusão de todos os grupos sociais no seu atendimento, existe a possibilidade de criação de novos equipamentos sociais, para atender às demandas advindas da incorporação de novas populações. Citamos, como exemplo, a criação de Centros e Unidades de Reabilitação, Centros de Atenção Psicossocial, Hospitais-Dia, Centros de Referência de Saúde do Trabalhador, Centros de Referência em Aids, Centros de Referência em Saúde da Criança, Centros de Convivência e Cooperativas, equipes de saúde mental e de reabilitação nas Unidades Básicas de Saúde, entre outros serviços (LEÃO et al., 2000).

Podemos citar a política da cidade de São Paulo, na gestão de 1989 a 1992, como uma experiência que buscou a efetivação do SUS e, para tanto, a inserção das áreas de saúde mental e saúde física no seu escopo de atendimento. Foram criadas equipes interdisciplinares que contavam com psicólogos, fisioterapeutas, fonoaudiólogos, educadores, médicos, enfermeiros e terapeutas ocupacionais, entre outras categorias, que operacionalizavam ações básicas de saúde (MALFITANO; LOPES, 2003). Como exemplos de ações, houve a implantação de grupos com pessoas com transtorno 
mental grave em Unidades Básicas de Saúde, atendimento individual e em grupo para portadores de deficiência, grupos terapêuticos e de geração de renda em Unidades Básicas de Saúde, ações nos Centros de Convivência e Cooperativas, além de muitas outras intervenções (OLIVER et al., 2005).

Em conjunto com o funcionamento de novas instâncias políticas, a população dita "excluída" do sistema passa a ter o direito de reivindicar uma assistência em saúde até então desconhecida. A base sólida para essa mudança é explicitada nos princípios e diretrizes do SUS, em que a atenção integral à saúde é dever do Estado e direito de todos os cidadãos. Como consequência ocorre, simultaneamente, uma abertura no campo de ação da terapia ocupacional, comprovando a intensa relação entre as possibilidades de atuação da profissão e as políticas públicas vigentes. $\mathrm{Na}$ experiência paulistana, a expansão da profissão é explicitada pela contratação de novos profissionais, via concurso público, que passaram de três, em 1989, para 241, no ano de 1992 (OLIVER et al., 2005).

Vale destacar a criação, naquele momento, de um equipamento inovador denominado Centro de Convivência e Cooperativa (CeCCo), que visava inserir e, principalmente, integrar grupos frequentemente discriminados, juntamente à população nomeada de "normal". Essa convivência ocorria por meio de oficinas, sendo o terapeuta ocupacional uma "peça-chave" para esse trabalho. Os CeCCos constituíramse como um exemplo da entrada de novos atores sociais no campo da saúde, com o desenvolvimento de ações em uma nova cena e em um novo espaço. A terapia ocupacional e seus fundamentos teóricos vão ao encontro daquela proposta, na medida em que visam intervir na área social de maneira a estabelecer a convivência entre as populações, a fim de proporcionar maior qualidade de vida e fomentar a cidadania (LOPES et al., 2002).

A partir de um novo ideário de saúde, os terapeutas ocupacionais têm a possibilidade de inserção profissional e implementação de intervenções, em novos e velhos espaços, que ofertem acesso aos grupos com quem trabalham. Verifica-se então que a inserção desse profissional não se dá isoladamente, de forma independente, apenas com o objetivo de atender determinada demanda, e sim que o tipo de população incluída pelas políticas de saúde determinará a necessidade ou não da inclusão da profissão no sistema público de saúde.

\section{Promoção e reorganização da Atenção Básica como estratégia para o fortalecimento do SUS}

Nesse controverso cenário, por um lado, de princípios legislativos abrangentes, práticas que demonstram, efetivamente, sua possibilidade e, por outro lado, a priorização de uma vertente econômica de enxugamento do Estado, é tecida a atenção em saúde no Brasil.

A estrutura de atendimento, com base nos princípios da regionalização e da hierarquização dos serviços, planeja uma gama de equipamentos sociais que se destinem à prestação de serviços em saúde a partir do critério da localização geográfica e da população abrangida, formando os Distritos de Saúde. Nestes, a porta de entrada do sistema ocorre pela Atenção Básica, que deve buscar o atendimento para, em média, $80 \%$ das demandas da população local (COHN; ELIAS, 1996).

Como estratégia para efetivar o ideário de atendimento na Atenção Básica, desde 1994 o Ministério da Saúde vem implantando, com crescimento contínuo por todo o país, a Estratégia de Saúde da Família. Inspirada no modelo britânico, como porta de entrada para o acesso ao sistema de saúde, centra-se na ação de um médico generalista, responsável por uma população determinada: o médico de família. Baseia-se também em experiências bem-sucedidas no Ceará e na Paraíba, a partir de 1990, com a implantação de programas que utilizam uma pessoa da comunidade para a promoção de ações de saúde, o denominado 'Agente Comunitário de Saúde' (BRASIL, 2001).

Seu crescimento e progressivo uso como estratégia da política brasileira de saúde para promoção e reorganização da atenção básica têm trazido o questionamento sobre os tipos de atendimentos que podem ser e são realizados, bem como as possibilidades e os limites de ampliação da demanda e atenção a todos os grupos populacionais presentes no território em que está inserido.

Como parte dessa discussão, propomos a diferenciação entre ação de saúde básica e ação básica de saúde, na defesa de que as ações básicas de saúde incorporam e ampliam o atendimento para todos, em uma perspectiva dada pelas demandas territoriais:

prevê ações de saúde básica, referindo-se a intervenções que digam respeito ao oferecimento de assistência para problemáticas ou doenças relacionadas aos componentes da saúde clínica, tais como: diabetes, hipertensão, prénatal, dentre outras. Para tais ações está previsto como equipe o agente comunitário, o enfermeiro, o auxiliar de enfermagem e o médico generalista. Já as ações básicas de saúde estão relacionadas com as demandas daquela população, de acordo com suas características socioculturais e econômicas. Trata-se de um outro conceito de saúde, que não se restringe à ausência de doença, pois tem como desafio a integração de toda a população no território e a atenção a essa população (MALFITANO; LOPES, 2003, p. 113). 
Embora seja de notória relevância o desenvolvimento das ações de saúde básica, para o controle epidemiológico de doenças e avanço nos índices de saúde da população, se houver uma restrição a essa dimensão, esta acaba por diminuir suas potencialidades de desenvolvimento mais pleno da proposição da atenção básica e contribui pouco no necessário avanço, no imaginário cultural, sobre o conceito de saúde e a ampliação da discussão da promoção da saúde, da qualidade de vida e da integração social (MALFITANO, 2007).

Atualmente, na tentativa de avançar na direção de suas potencialidades e ampliação de seu atendimento, segundo os princípios e diretrizes previstos no SUS, o Ministério da Saúde aprovou a Portaria 154 (BRASIL, 2008), que cria os:

Núcleos de Apoio à Saúde da Família - NASF com o objetivo de ampliar a abrangência e o escopo das ações de atenção básica, bem como sua resolubilidade, apoiando a inserção da estratégia de Saúde da Família na rede de serviços e o processo de territorialização e regionalização a partir da atenção básica (Artigo $1^{\circ}$.).

Os NASFs projetam a inserção das seguintes intervenções: Atividade Física/Práticas Corporais, Práticas Integrativas e Complementares (Acupuntura e Homeopatia), Reabilitação, Alimentação e Nutrição, Saúde Mental, Serviço Social, Saúde da Criança, Saúde da Mulher e Assistência Farmacêutica (BRASIL, 2008, Anexo I). A Portaria determina ainda que o NASF deve ser composto por, no mínimo, três ou cinco profissionais de nível superior (a depender da extensão e população do município), listando, dentre eles, o terapeuta ocupacional (Incisos $2^{\circ}$. e $4^{\circ}$. Artigo $3^{\circ}$.).

Há, portanto, uma legislação recente que vislumbra o crescimento das equipes de atenção básica para a ampliação de suas ações e com demanda de recursos humanos para tais trabalhos. Aponta, consequentemente, o desafio para os terapeutas ocupacionais, de ocuparem esses cargos, que potencialmente serão criados, e intervirem para o fomento de ações com os grupos-alvo para os quais dedicam sua atenção, ampliando o acesso daquelas pessoas e seu exercício ao direito à saúde.

\section{O encontro dos princípios}

À terapia ocupacional foram ofertados novos espaços de intervenção com a implantação do SUS, pois, além da necessidade de um profissional para atender grupos populacionais específicos, como as pessoas com transtorno mental e/ou com deficiência física, esta categoria profissional desenvolve sua ação pautada nas necessidades apresentadas pelo usuário, como na realização de oficinas terapêuticas, nas quais as atividades são conduzidas por este princípio. Esse fato vai ao encontro dos princípios do SUS, que consideram as várias dimensões do processo saúde-doença, a fim de garantir a promoção, proteção, cura e reabilitação dos indivíduos e do coletivo. O sistema preconiza a implementação de ações coerentes com a demanda da população, tanto pela procura dos serviços, quanto pelos estudos de adscrição territorial, que revelam o perfil social e epidemiológico de determinada região.

Distanciando-se da lógica do modelo médicoassistencial-privatista, a profissão desenvolve-se em direção ao tratamento do ser humano de forma integral, com uma ação voltada para as necessidades sociais de saúde sob a perspectiva da integralidade, caminhando para o desenvolvimento de ações que vão além dos princípios reducionistas médico-organicistas. Torna-se um desafio político, com características motivadoras, distanciar-se da lógica do capital, não priorizando "apenas", no sistema público, as necessidades reconhecidas como não lucrativas, respondendo positivamente à atenção universal e, todavia, ao direito à saúde para todos os cidadãos brasileiros.

Entre os princípios doutrinários do SUS, destaca-se a integralidade como principal característica comum entre o que é pretendido pelo sistema de saúde e a forma de atuação dos terapeutas ocupacionais. Durante a formação profissional é sempre ensinada e destacada aos alunos a importância da incorporação e intervenção nas dimensões biológicas, psicológicas e sociais, demonstrando a necessidade de ações que partam da integralidade como fundamento. Vislumbrar os sujeitos em sua integralidade significa compreendê-los em seu contexto socio-histórico-cultural e econômico, admitindo-o como um ser biopsicossocial, buscando, por meio do acolhimento e da escuta qualificada (condutas preconizadas pelo SUS), identificar as reais necessidades do indivíduo de forma a propiciar a ele a possibilidade de exercício de seus direitos e, com isso, a qualidade de vida de forma continuada.

Vasconcelos e Pasche (2007) resumem a idéia do princípio organizativo da integralidade:

Esse princípio orientou a expansão e qualificação das ações e serviços de saúde do SUS que ofertam desde um elenco ampliado de imunizações até os serviços de reabilitação física e mental, além das ações de promoção da saúde de caráter intersetorial (p. 535).

A ação da terapia ocupacional no SUS, juntamente com suas intervenções no campo da educação e na esfera social, fortalece cada vez mais a profissão, que tem na sua identidade princípios condizentes ao SUS. Revela-se uma 
atuação profissional que congrega aspectos tecno-científicos e humanitários, nos quais todos são beneficiados: a equipe, o indivíduo, o coletivo e também o próprio sistema. A terapia ocupacional, ao atender às necessidades dos sujeitos, trabalha para a inserção social daqueles com quem intervém, levando em consideração as reais possibilidades individuais e coletivas, objetivando a promoção da autonomia e da independência para incorporação e participação ativa na sociedade.

\section{CONSIDERAÇÕES FINAIS}

Faz-se necessária uma reflexão crítica sobre o discurso da "ineficiência" do SUS, distinguindo-se sobre qual aspecto se aborda essa generalidade, assim como as causas e os interesses intrínsecos a este quadro. A conjuntura hegemônica econômica cria lacunas importantes no desenvolvimento do SUS e, com isso, deixa incompletudes e desassistências na atenção pública em saúde aos cidadãos brasileiros.

Entretanto, sob um olhar histórico, as conquistas reconhecidas pela Carta Constituinte são de absoluta relevância e demonstram um processo recente de aquisição de direitos e passagem do lugar do assistencialismo e da caridade para a esfera pública de atendimento.

Esses fatos marcam a história do desenvolvimento da profissão terapia ocupacional no Brasil, uma vez que colocam, lentamente, populações alijadas da participação social na arena sociopolítica e, por conseguinte, trazem a terapia ocupacional, assim como outras profissões, do lugar de profissão "caritativa" para a necessidade de desenvolvimento de tecnologias de cuidado e assistência, que respondam às demandas de tais grupos populacionais.

A institucionalização da terapia ocupacional, dada em seu início, resumidamente, pelos aspectos da ocupação como forma de tratamento dos doentes mentais nos manicômios e da reabilitação dos incapacitados físicos nos hospitais do Exército, modifica-se na medida em que passa a ter a função social de contribuir para legitimar as diretrizes constitucionais dos direitos sociais. Evoluindo de seu histórico enraizado em ações tidas como assistencialistas e caritativas para o novo contexto dos direitos sociais que focalizamos aqui na discussão das políticas de saúde, a profissão ganha novos espaços de ação, contribuindo para implementar efetivamente as políticas sociais e, portanto, o SUS, o que, consequentemente, amplia o desenvolvimento da categoria.

As ações sociais, incluindo os serviços de saúde, são realizadas por sujeitos que, no seu poder local, ampliam ou retraem o atendimento e criam metodologias para realizá-lo para determinada população. Assim, trata-se de um papel técnico e político do terapeuta ocupacional acompanhar as discussões em curso, posicionar-se e intervir em prol do acesso aos direitos reconhecidos daqueles com quem atua, tendo como horizonte a participação e inserção social de todos, almejando o projeto de uma sociedade menos desigual e mais solidária.

MALFITANO, A. P. S., FERREIRA, A. P. Public health and occupational therapy: appointments on current and historical relations. Rev. Ter. Ocup. Univ. São Paulo, v. 22, n. 2, p. 102-109, maio/ ago. 2011.

\begin{abstract}
It is affirmed that Brazilian Public Health policies have rooting and influence in the social-historical development of Occupational Therapy profession. So, this study starts from the historical-political course of these two fields and their establishing as institutions, in a route from philanthropy to social rights, acheived due to brazilian democratization movement, which took place in the end of 1980's and enlarged social rights for everyone. One of its results was the implementation of Sistema Único de Saúde (Health Public System), which provides universal care, including groups that were, up to that time, attended mainly by philanthropic services. This need has created a lack of professionals which were, in that period, scarce in the public health system, as Occupational Therapists. From that time on, technicians have created new services, and care and attention technologies which have made possible the yet precarious insertion of some groups into the access to the health system and, consequently, the development of that profession. So, it is pointed that through the establishing of public policies which expand the access to social rights of the groups targeted by Occupational Therapy, it is possible, as a consequence, to enlarge professional intervention fields and professional development. Finally, the current difficulties around the political-economical model and the permanent challenge of enlarging attention and inclusion of groups into social rights access are pointed out.
\end{abstract}

KEY WORDS: Occupational therapy; Public policies; Unified Health System; Right to health. 


\section{REFERÊNCIAS}

BASAGLIA, F. O Homem no Pelourinho. Educação e Sociedade, n. 25, p. 73-95, 1986.

BRASIL. Ministério da Saúde. Secretaria Nacional de Assistência à Saúde. $A B C$ do SUS: doutrinas e princípios. Brasília: Imprensa Oficial, 1990.

BRASIL. Ministério da Saúde. Secretaria Executiva. Programa Saúde da Família. Brasília: Ministério da Saúde, 2001.

BRASIL. Ministério da Saúde. Portaria GM No. 154. Cria os Núcleos de Apoio à Saúde da Família - NASF. Diário Oficial da União, Brasília, DF, n. 18, p.47-49, 25 jan 2008.

CARVALHO, S. R. O Estado e a democracia em tempos neoliberais: novos desafios para o Sistema Único de Saúde. Mimeo, 2000.

COHN, A.; ELIAS, P. M. Saúde no Brasil: políticas e organização dos serviços. São Paulo: Cortez/CEDEC, 1996. p. 11-55.

DE CARLO, M. M. R. P.; BARTAlOTTI, C. C. Caminhos da terapia ocupacional. In: DE CARLO, M. M. R. P.; BARTALOTTI, C. C. (Org.). Terapia ocupacional no Brasil: fundamentos e perspectivas. São Paulo: Plexus, 2001. p. 19-40.

ESCOREL, S. Elementos para análise da configuração do padrão brasileiro de proteção social - o Brasil tem um Welfare State? Cadernos Fiocruz, Série Estudos, $n^{\circ} 1$, Política, Planejamento e Gestão em Saúde, 1993.

FOUCAULT, M. Microfisica do poder. Rio de Janeiro: Graal, 1979.

LEÃO, A. et al. Terapia ocupacional e políticas públicas de saúde na cidade de São Paulo. Cadernos de Terapia Ocupacional da UFSCar, v. 8, n. 1, p. 48-56, 2000.

LOPES, R. E.; LEÃO, A. Terapeutas ocupacionais e os centros de convivência e cooperativas: novas ações de saúde. Revista de Terapia Ocupacional da Universidade de São Paulo, v. 13, n.2, p. 56-63, 2002.

MALFITANO, A. P. S. Atrás da porta que se abre: demandas sociais e o Programa de Saúde da Família. Holambra, SP: Setembro, 2007.

MALFITANO, A. P. S.; LOPES, R. E. Programa de Saúde da Família e agentes comunitários: demandas para além da saúde básica. Revista de Terapia Ocupacional da Universidade de São Paulo, v. 14, n. 3, p.110-117, 2003.

MENDES, E. V. Distrito sanitário: o processo social de mudança das práticas sanitárias do Sistema Único de Saúde. São Paulo, Rio de Janeiro: Hucitec/Abrasco, 1993.

NASCIMENTO, B. A. Trabalho, loucura e ordem: o uso do trabalho e da ocupação em instituições psiquiátricas. 149p. Dissertação (Mestrado). Pontifícia Universidade Católica de São Paulo. São Paulo, 1991.

OLIVER, F. C.; BARROS, D. D.; LOPES, R. E. Estudo sobre a incorporação da terapia ocupacional no contexto das ações de saúde mental e saúde da pessoa com deficiência no Município de São Paulo entre 1989 e 1993. Revista de Terapia Ocupacional da Universidade de São Paulo, v. 16, n. 1, p. 31-39, 2005.

PAIM, J. S. Modelos de atenção e vigilância da saúde. In: ROUQUAYROL, M. Z.; ALMEIDA FILHO, N. Epidemiologia e saúde. 5a ed. Rio de Janeiro: MEDSI, 1999. p. 567-573.

SOARES, L. B. T. História da terapia ocupacional. In: CAVALCANTI, A., GALVÃO, C. (Orgs.). Terapia ocupacional: fundamentação e prática. Rio de Janeiro: Guanabara Koogan, 2007. p. 3-9.

VASCONCELOS, C. M.; PASCHE, D. F. O Sistema Único de Saúde. In: CAMPOS, G. W. S. et al. Tratado de saúde coletiva. São Paulo: Hucitec/Fiocruz, 2007. p.531-562.

VERDĖS-LEROUX, J. Trabalhador social: prática, hábitos, ethos, formas de intervenção. São Paulo: Cortez, 1986. 